\title{
EXISTENCE OF NON-RADIALLY SYMMETRIC VISCOSITY SOLUTIONS TO SEMILINEAR DEGENERATE ELLIPTIC EQUATIONS WITH RADIALLY SYMMETRIC COEFFICIENTS IN THE PLANE, PART II
}

\author{
KENJI MARUO AND NAOKI YAMADA
}

Abstract. We study continuous viscosity solutions for a semilinear degenerate elliptic equation with radially symmetric coefficients in the plane. If the equation satisfies certain relations with respect to the behavior of coefficients at the infinity, then it is known that there exist many solutions. Our purpose is to construct many non radially symmetric solutions satisfying the similar behavior with radial symmetric solutions at the infinity. The solutions are obtained as a small perturbation from a radially symmetric solution. We construct super- and sub-solution by using the series expansion of $r^{\alpha-j \beta} \cos n \theta(j, n=1,2, \ldots)$, where $(r, \theta)$ is the polar coordinate and $\alpha$ and $\beta$ are certain positive constants.

Mathematics subject classification (2010): 34B40, 35D05, 35J60, 35J70.

Keywords and phrases: non-radially symmetric solution, degenerate elliptic equation, semilinear elliptic equation, continuous viscosity solution.

\section{REFERENCES}

[1] M. G. CRAndall, H. Ishit And P. -L. Lions, User's guide to viscosity solutions of second order partial differential equations, Bull. Amer. Math. Soc., 27 (1992), 1-67.

[2] S. KoIKE, A Beginner's Guide to the Theory of Viscosity Solutions, MSJ Memoirs 13, Math. Soc. Japan, 2004.

[3] D. Gilberg And N. S. Trudinger, Elliptic Partial Differential Equations of Second Order, SpringerVerlag, 1983.

[4] K. MARUO AND N. YAMADA, Existence of non-radially symmetric viscosity solutions to semilinear elliptic equations with radially symmetric coefficients in the plane, Part I, J. Math. Anal. Appl., 345 (2008), 743-753.

[5] K. Maruo And Y. Tomita, Radial viscosity solutions of the Dirichlet problem for semilinear degenerate elliptic equations, Osaka J. Math., 38 (2001), 737-757.

[6] K. MARUO AND Y. TOMITA, Unbounded radially symmetric viscosity solutions of semilinear degenerate elliptic equations, Sci. Math. Japonicae, 58 (2003), 107-123.

[7] E. S. NOUSSAIR AND C. A. SWANSON, Positive solution of quasilinear elliptic equations in exterior domains, J. Math. Anal. Appl., 75 (1980), 121-133. 